Acknowledgment. The author wishes to thank Professor H. G. Elrod, Jr. for suggesting the problem.

Columbia University

New York, New York

1. G. N. Watson, A Treatise on the Theory of Bessel Functions, 2nd ed., Cambridge Univ. Press, New York, 1944. MR 6, 64.

2. R. S. Burington, Handbook of Mathematical Tables and Formulas, 3rd ed., Handbook Publishers Inc., Sandusky, Ohio, 1949. MR 22 \#2514.

\title{
Footnote to the Evaluation of Certain Complex Elliptic Integrals
}

\section{By C. D. Sutherland}

The formulas for evaluating the elliptic integral of the third kind wi $h$ a complex parameter as given by Byrd and Friedman [1] have been corrected and simplified Iby Lang and Stevens [2]. There is, however, a further correction necessary in these latter results.

The integral to be evaluated is

$$
I=\left(a_{1}+i b_{1}\right) \int_{0}^{\phi} \frac{d \theta}{\left(1-\alpha^{2} \sin ^{2} \theta\right) \Delta},
$$

where $\alpha^{2}$ is complex and $\Delta=\sqrt{ }\left(1-k^{2} \sin ^{2} \theta\right)$. In the formulas for evaluating $I$ there appears the quantity

$$
\tau_{2}=\int_{0}^{p_{2}} \frac{m_{2} d x}{1+h_{2} x^{2}}=\frac{m_{2}}{\sqrt{ } h_{2}} \tan ^{-1}\left(p_{2} \sqrt{ } h_{2}\right)
$$

where

$$
p_{2}=\frac{\sin \phi \cos \phi}{\left(1+m_{2} \sin ^{2} \phi\right) \Delta} .
$$

We will consider the case where $m_{2} \leqq-1$. If this occurs we see that as $\phi$ goes to $\pi / 2$, either $p_{2} \rightarrow \infty\left(m_{2}=-1\right)$ and $\left[\tan ^{-1}\left(p_{2} \sqrt{ } h_{2}\right)\right] \rightarrow \pi / 2$, or $p_{2} \rightarrow 0$ through negative values $\left(m_{2}<-1\right)$ and $\left[\tan ^{-1}\left(p_{2} \sqrt{ } h_{2}\right)\right] \rightarrow \pi$ (and not to zero). To avoid overlooking this possibility the proper representation for $\tau_{2}$ is

$$
\begin{aligned}
\tau_{2} & =\frac{-1}{\sqrt{ } h_{2}} \cos ^{-1}\left(\frac{\Delta \cos \phi}{\sqrt{ }\left(h_{2} \sin ^{2} \phi+\Delta^{2} \cos ^{2} \phi\right)}\right) \text { for } m_{2}=-1 \\
\tau_{2} & =\frac{m_{2}}{\sqrt{ } h_{2}} \cos ^{-1}\left(\frac{\Delta\left(1+m_{2} \sin ^{2} \phi\right)}{\sqrt{ }\left(h_{2} \sin ^{2} \phi \cos ^{2} \phi+\Delta^{2}\left(1+m_{2} \sin ^{2} \phi\right)^{2}\right)}\right) \text { for } m_{2} \neq-1 .
\end{aligned}
$$

It is to be noted, in particular, that the formulas for the real and imaginary parts of the complete integral should contain a term involving $\tau_{2}$ whenever $m_{2} \leqq-1$.

Received March 16, 1964. Work performed under the auspices of the U. S. Atomic Energy Commission. 
Equation $\left(416.00^{\prime}\right)$ in reference [2] and equations $(416.00)$ to $(419.00)$ in reference [1] do not contain this term and should be changed.

University of California

Los Alamos Scientific Laboratory

Los Alamos, New Mexico

1. PaUl F. Byrd \& Morris D. Friedman, Handbook of Elliptic Integrals for Engineers and Physicists, Die Grundlehren der Mathematischen Wissenschaften in Einzeldarstellungen, Bd. LXVII, Springer, Berlin, 1954. MR 15, 702.

2. H. A. LAN \& D. F. StEvens, "On the evaluation of certain complex elliptic integrals," Math. Comp., v. 14, 1960, p. 195-199. MR $22 \# 3095$.

\title{
On Davis' Method of Estimating Quadrature Errors
}

\author{
By Y. T. Lo, S. W. Lee, and B. Sun
}

In quadrature method the error is traditionally estimated in terms of the high derivatives of the integrand. The drawbacks of this method are well known. Some ten years ago, Davis [1], Davis and Rabinowitz [2] introduced an interesting new method in estimating the error for analytic functions in terms of their norms. Briefly, for any function $f(z)$ belonging to $L^{2}\left(\varepsilon_{p}\right)$, where $\varepsilon_{p}$ is a region in the complex $z$-plane, bounded by an ellipse with foci at $(-1,0)$ and $(1,0)$, the error $E$ associated with the quadrature

$$
\int_{-1}^{+1} f(x) d x=\sum_{k=0}^{N} a_{k} f\left(\lambda_{k}\right)+E(f)
$$

is bounded by

$$
|E(f)| \leqq \sigma_{R}\|f\|_{\varepsilon_{p}}
$$

In the above relation,

$$
\|f\|_{\varepsilon_{p}}=\left[\iint_{\varepsilon_{p}}|f(z)|^{2} d x d y\right]^{1 / 2}
$$

and $\sigma_{R}$ depends only on the ellipse $\varepsilon_{p}$ and the quadrature rule $R$.

Davis and Rabinowitz [2] have given a short table of $\sigma_{R}$ for a few commonly used quadrature rules and various values of the semi-major axis $a$. To test their results, we have assumed for $f$ a simple trigonometric function whose integral can be easily evaluated and computed. By comparing this with those obtained by various quadratures it turns out that the actual errors are larger than the bound $\sigma_{R}\|f\|_{\varepsilon_{p}}$. Thus it leads us to believe that their tabulated values of $\sigma_{R}$ are in error. In a private communication, Davis agreed with us and encouraged us to recompute their table. Recently we completed this task. The results are tabulated below, where a few more cases and a wider range in semi-major axis $a$ are included. To our disappointment, it is found that these values are much greater than theirs, nearly by a factor of 4 .

Received May 11, 1964. 